\title{
Identifying accessibility conditions for children with multiple disabilities: a Virtual Reality Wheelchair simulator
}

\author{
Nancy Rodriguez* \\ LIRMM - University of Montpellier
}

\begin{abstract}
Training is one of the main domain applications of Virtual Reality (VR). Simulation and visual realism provide training situations very close to practice with real systems but at reduced cost and with greater safety. Furthermore VR offers the possibility of change time or space scales, visualize from different perspectives, experience inaccessible real environments, under the control of the user, without risks, progressing at her own pace. This allows to develop skills and to have confidence to work in a later stage in real conditions with real equipment.

Many interaction technologies are now more widely available and affordable. But generally devices are conceived for "standard" people leaving behind people with impairments and further accentuating the digital gap. In this paper, we present our work in the development of an accessible wheelchair simulator designed to allow children with multiple disabilities to familiarize themselves with the wheelchair, and practitioners to better understand children capabilities.
\end{abstract}

Keywords: virtual reality, simulator, disability, multiple disabilities, wheelchair, learning, augmented and alternative communication, interaction devices

Index Terms: I.3.1 [Computer Graphics]: Hardware Architecture - Input devices; I.3.7 [Computer Graphics]: Three-Dimensional Graphics and Realism - Virtual reality; H.5.1 [Information Interfaces And Presentation]: Multimedia Information Systems Artificial, augmented, and virtual realities H.5.2 [Information Interfaces And Presentation]: User Interfaces - Input devices and strategies

\section{INTRODUCTION}

Virtual Reality is used for medical applications as phobias treatment or in rehabilitation to develop physical skills in a playful way. Indeed, 3D environments provides users with the sense of control, they can stop the experience it if the situation is difficult, repeat a session as many times as needed and evolve at their own pace. With a simulation, it is possible Erreur ! Source du renvoi introuvable.Erreur ! Source du renvoi introuvable.. Moreover, for practitioners, the virtual environment coupled to a simulation and analysis tool, allows to create and adapt experiences to different users, with different motion and cognition skills. Erreur ! Source du renvoi introuvable.

The objective of this paper is to present the design and development of an accessible wheelchair simulator for children

* nancy.rodriguez@lirmm.fr with multiple disabilities, aged between 13 and 16 years. Learning to drive a wheelchair in real conditions can be dangerous, tiresome and even scary. This simulator will prepare children to wheelchair driving, helping them to manage the emotions triggered by this activity because it represents a very big step in autonomy and freedom.

"Multiple disabilities" means simultaneous impairments (intellectual disability, orthopedic impairment, sensory deficits, health or neurological disorders), the combination of which causes such severe communication and other developmental and educational needs. Children with multiple disabilities require support in major life activities and specialized instruction due to limited communication capabilities, a tendency to forget skills through disuse, a high sensibility to failure and trouble generalizing skills from one situation to another.

For these reasons, this project is carried out in collaboration between the Institute for Children and Teenagers with multiple disabilities Coste Rousse located in Prades-le-Lez (Hérault, France) and the Laboratory of Informatics, Robotics and Microelectronics of Montpellier (LIRMM) in Montpellier. We work closely with the multidisciplinary team (educators, orthoptist, physiotherapist) of the center to make the simulator accessible from this conception and to assess children interest in this kind of IT tool.

\section{Related Work}

Several wheelchair simulators are available, plenty of realism effects and good ideas to learn to drive, evaluate buildings accessibility and to allow others to understand the difficulties of using a wheelchair [4-8]. However, there are mainly designed for people with only motor impairment. There is not, to our knowledge, a simulator designed or adaptable for children with multiple disabilities. Generally, these systems have realistic sets with many details and animations. This type of scene is not suitable for children suffering from cognitive disorders. Indeed, the details used to increase the realism and immersion of the user will cause difficulties in perception and understanding of the environment.

\section{DESIGN}

The simulator is developed under Unity3D, a game engine allowing to create and manage interactive virtual environments. Unity offers several functionalities such as a physics engine, a tool for build animations and the possibility to easily integrate many input and output devices which is the most important feature for our work.

VR simulation is frequently associated with high realism and immersion. In our case, these elements must be used with restraint because a high level of details cause difficulties in children perception and disturb the analysis of significant elements. Our scenes (textures and light) must be then very clean. In the same direction, "gamification" wouldn't go so far, for example, there is 


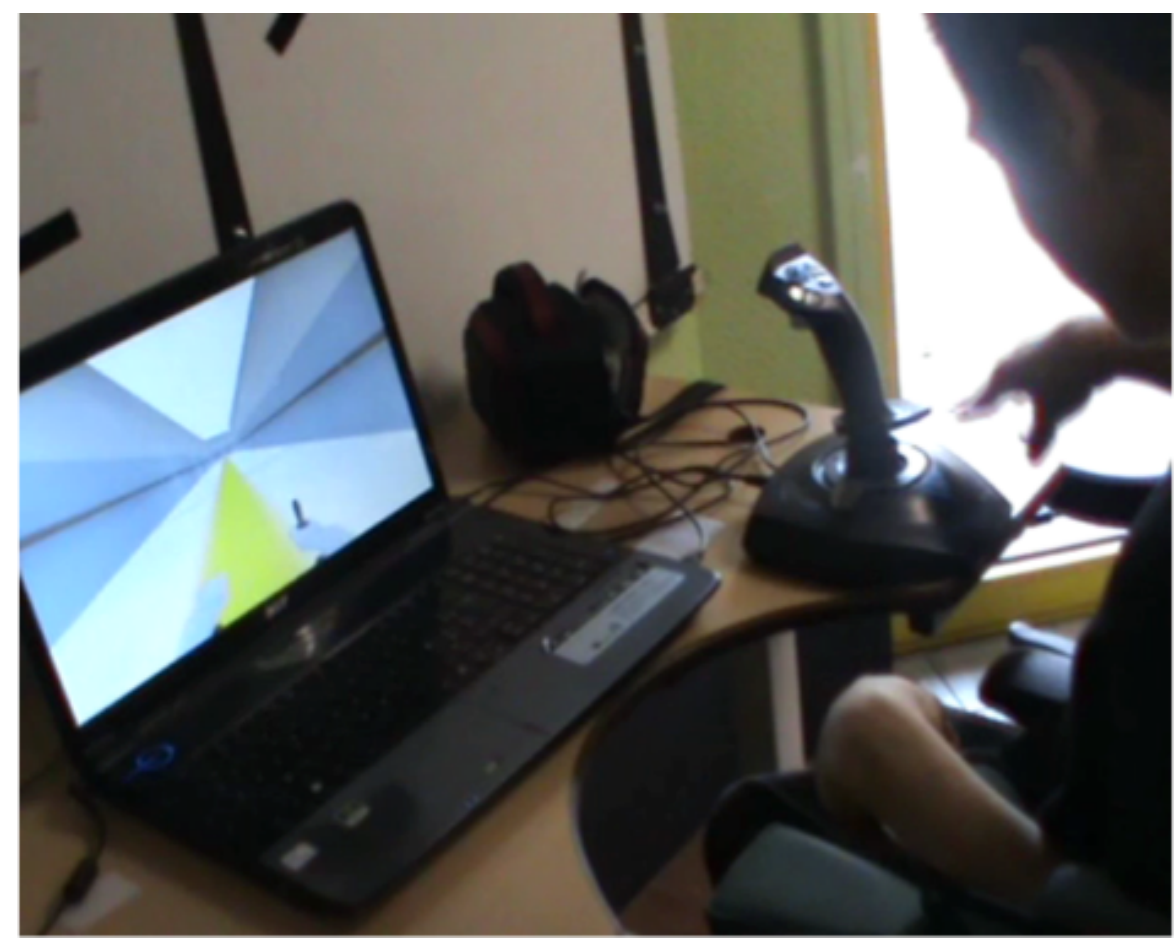

Figure 1. Test of the wheelchair simulator

not timer and neither "failure" feedback (only positive reinforcement).

Besides the simulation, we have also designed and implemented a scene editor, allowing the creation of new environments to be used within the simulator.

\section{INTERACTION DEVICES}

Technology evolves very quickly and has transformed the way we interact with computers including touch and gestures, for example the "sweep movement" used in smartphones or tablets. But devices are conceived for "standard" people leaving behind people with impairments and further accentuating the digital gap. Indeed people with severe motor dysfunction can have muscles too weak to wear a device or not fine enough motor skills, difficulties to raise an arm and keep the pose or very slight differences between voluntary and involuntary movements. If we add cognition troubles, it is clear that we need to provide adapted interfaces.

Mouse or traditional joysticks are not adapted to our users. We have then add support for adapted joysticks (joystick from a wheelchair adapted to be connected to the computer using a usb port), contactors and eye-trackers. This allows addressing varied communication capabilities in order to have an accessible simulation. In addition, this feature may help find which devices are suitable for a particular user.

\section{Evaluation}

Even if we want to foster acceptability by including the user from the conception, it is clear we cannot directly interact with our final users. Application bugs of an early prototype will provoke frustration and moreover rejection. In addition, we cannot work with the children regularly. For these reasons, development and evaluation of our system advance slowly.
During the test sessions we observed a lot of curiosity about the simulator and different ways of apprehend it: one children focus on the screen and try to use the joystick, two children watch the screen but doesn't want to work with it, one children was easily distracted by our presence.

One important element is to evaluate our system and to know if skills will be translate into the real world because we cannot use traditional VR evaluations based on questionnaires and assessment of the experience by subjects themselves. To overcome this issue, we have developed a "replay" system allowing to observe the camera changes and the itinerary of the user during the simulation. We will use this new feature in our next tests stages.

\section{Conclusion}

We presented here a description of our work concerning the design, implementation and evaluation of a virtual reality wheelchair simulation for children with multiple disabilities. Our tests have showed that children are interested and able to use the simulator in its last version. Practitioners, relatives and adults with motor impairment are also interested (a multiuser feature has been recently added). The simulator is again included this year in the learning program of several children at the Coste Rousse center.

\section{ACKNOWLEDGEMENTS}

I would like to thank the staff of Coste Rousse center and all the students who had participate to the development of the simulator.

\section{REFERENCES}

[1] D. Mellet-d'Huart and G. Michel. Les environnements Informatiques pour l'Apprentissage Humain. Hermes, 2006, ch. 11, "Réalité virtuelle et apprentissage". 
[2] D. Le Gall and P. Allain. Applications des techniques de réalité virtuelle à la neuropsychologie clinique. Champ psychosomatique, no 22, p. 25-38 (2001).

[3] M. Arpaillance and A. Debrill : Évaluer la communication de la personne polyhandicapée. Master's thesis, Université de Lille, Droit et Santé, 2013.

[4] L. Marchal-Crespo. A simulator and assist-as-needed control strategy for learning to drive a power wheelchair. $\mathrm{PhD}$ thesis, University of California, Irvine, 2006.

[5] N. Steyn. Virtual Reality Platform Modelling and Design for Versatile Electric Wheelchair Simulation in an Enabled Environment. PhD thesis, Department of Electrical Engineering, Tshwane University of Technology, 2014.

[6] A. Schmid and C. Dumas. Accessim, 2008-2013, http://www.accessim.ceremh.org/index.html

[7] Y. Morère. VIEW : Virtual electric wheelchair, 2012, http://www.lasc.univ-metz.fr/spip.php?article41

[8] LifeTool. Wheelsim. http://www. lifetool-solutions.at

[9] C.K. Olson. Children's Motivations for Video Game Play in the Context of Normal Development. Review of General Psychology, 2010

[10] C. Pinheiro Jr, E. Naves, P. Pino, E. Losson, A. Andrade and G. Bourhis. Alternative communication systems for people with severe motor disabilities: a survey. BioMedical Engineering OnLine, 2011 